\title{
Socio-ethical Dimension of COVID-19 Prevention Mechanism-The Triumph of Care Ethics
}

\section{Charles Biradzem Dine ${ }^{1}$}

Received: 26 May 2020 / Revised: 27 July 2020 / Accepted: 30 July 2020 /

Published online: 12 August 2020

(C) National University of Singapore and Springer Nature Singapore Pte Ltd. 2020

\begin{abstract}
The psycho-social day-to-day experience of COVID-19 pandemic has shone some light on the wider scope of health vulnerability and has correspondingly enlarged the ethical debate surrounding the social implications of health and healthcare. This emerging paradigm is neither a single-handed problem of biomedical scientists nor of social analysts. It instead needs a strategically oriented collaborative and interdisciplinary preventive effort. To that effect, this article presents some socio-ethical reflections underscoring the judicious use of the insight from care ethics as an asset in minimizing the possible propagation of the COVID-19 virus and the escalation of its vulnerability in the day-to-day human interaction. It further emphasizes that if this insight is overlooked, the effects of the diverse facets of the "shadow pandemics" of COVID19 - fallouts on both the affected and the infected - may equally be deadly.
\end{abstract}

Keywords Care ethics $\cdot$ COVID-19 pandemic $\cdot$ Vulnerability $\cdot$ Stigmatization

\section{Introduction-Socio-ethical Reflections Surrounding COVID-19}

The crisis resulting from COVID-19 has imposed various alterations in human daily routines in the whole world without any exception and has incited new thoughts and reflections about human daily life and interactions. At minimum, the pandemic has confirmed some fundamental realities that hitherto seemed ambiguous:

- The "sameness" of humanity/humankind that normally goes beyond various accidental properties of a human being like the colour of the skin, the language of expression and the socio-cultural background ${ }^{1}$

${ }^{1}$ See Humanity in Online Vocabulary Dictionary: https://www.vocabulary.com/dictionary/humanity. Accessed 18 May 2020.

Charles Biradzem Dine

Charles.biradzem.dine@umontreal.ca

1 Applied Human Sciences, University of Montreal, Montreal, Canada 
- The wider connotation of human health as "a state of complete physical, mental, and social wellbeing, and not merely the absence of disease or infirmity" (OHCHR and WHO 2008, 1)

- The multidimensional and wide-ranging inequality gap of health vulnerability in our societies

The impact of these ambiguities cannot be overlooked: (1) they are complicating the job of researchers to determine the degree and vector of vulnerability vis-à-vis COVID-19 so as to define effective preventive measures; and (2) various stakeholders concerned with the fight against COVID-19 need the updates on the alterations of vulnerability in order to better calibrate preventive mechanisms. Though vulnerability is purported a universal characteristic of every human being (de Groot et al. 2019), it is clearer that some groups or categories of people are more susceptible to the calamities of COVID-19 than others for varied reasons (Xafis 2020). But due to the aforementioned ambiguities, "the vulnerable" and "the asymptomatic status", in relation to COVID-19, are yet to be defined to be targeted/regulated, and victims abound.

However, it is a common phenomenon that at the level of preventive researchas it is still the case with COVID-19-multidisciplinary and interdisciplinary contributions replace single-discipline investigations (Scrimshaw et al. 2001). As part of that contribution, this article highlights some salient ideas deemed productive enough to enhance various preventive measures against COVID-19 pandemic, as well as against any human-to-human communicable pandemic. Given the general biopsychosocial dimensions of human health (Dine 2016), the article highlights some basic socio-ethical reflections - mainly from the trajectory of care ethicsthat could complement and boost the effectiveness of the preventive framework against COVID-19.

In fact, we are neither stepping into the field of biomedical sciences nor challenging it. We are instead complementing it. We argue that when facing a pandemic of this magnitude, the assessment of vulnerability should be taken beyond general/universal vulnerability to health harm and consider various specificities of the susceptibility and adaptability to the crisis in question as part of the prevention mechanism. However, some of our views might have been influenced by the COVID-19 experience in the Western world, and, particularly, in North America. By this we are acknowledging that as strategies for lockdown and confinement could not be straightforwardly universalized, so might also be responses to easing them. Definitely, specific support for vulnerable groups must necessarily be tailored to specific contexts and needs. Nonetheless, the analysis in this article is based on care ethics as an essential foundation/ complement for anti-COVID-19 ambitions.

\section{Care Ethics-Approach and Precision}

Though care ethics is relatively young as a concept (Held 2007), it is attracting much attention, especially among feminist scholars (Okano 2016). Nevertheless, it is also highly promoted in biomedical and healthcare ethics with much emphasis on empathy, believing that it activates trust and openness to understand and address the need of 
patients (van Dijke et al. 2019; De Panfilis et al. 2019; Nair 2005). But given the enlarged connotation of human health and healthcare, care ethics has come to be central in the socio-political, socio-cultural and socio-economic aspects of human life and wellbeing (Held 2007). This is why care ethics is still suffering from definition crisis as each school of thought or professional affiliation formulates its definition to suit their taste. However, for the sake of this article, the common features of care ethics retained are empathy (van Dijke et al. 2019), responsibility (Held 2007), attentiveness and responsiveness (Nair 2005).

In the ensemble, care ethics is simply understood as the ethics of the responsibility that ultimately manages the web of relationships that connect everyone for the good of everyone (Kroeger-Mappes 1994). From its main characteristics, care ethics directly contrasts some key philosophical tenets like deontological and consequential/utilitarian theories and inclines more to moral theories like those of Confucianism (Sander-Staudt 2020) and African communitarianism which are built on love and care for one another (Metz 2013). This makes it more of a philosophical virtue than a theory because

care ethics involves maintaining the world of, and meeting the needs of, ourself and others. It builds on the motivation to care for those who are dependent and vulnerable, and it is inspired by both memories of being cared for and the idealizations of self. (Sander-Staudt 2020)

If we just limit ourselves at this level, we can ascertain that care ethics is also-directly or indirectly - the ethics of moral duties and rights. As seen above, it is centred on the responsibility to understand and address the problems of each person in a web of social relationship motivated by empathy. In other words, it promotes and valorizes "altruism" by rendering the act of caring for/and about the wellbeing of others a moral responsibility (Kroeger-Mappes 1994). Since this does not signify that there is a particular group of persons designated to care for others than as an aspect of interrelational moral responsibility to care for each other, it means, in other words, that we irrespectively have the duty to care for fellow citizens. As social beings, this duty is necessarily complemented by the moral right to care from fellow citizens or society when need be.

In fact, "right", as referred here, has a moral connotation that meets with "duty" in "care" as benevolence that regulates our attitude towards each other (Kroeger-Mappes 1994). These two ends - duty and right — are brought together by "kindness" equated to humanity, since to be human is to be kind, compassionate and sympathetic, with generous behaviour ${ }^{2}$. The core of this approach is "care", "conceived both as an action concretely expressed towards the other, and as a value that has the goal of being universally shared, beginning with the awareness of the fragility and vulnerability of the human condition" (De Panfilis et al. 2019, 2). Seen in this way, care ethics provides us with an important and solid ethical basis for assessing and prioritizing the core ethical issues at stake with the COVID-19 pandemic. Key among these is to recognize/ identify COVID-19's fragility and vulnerability, so as to proficiently orientate our caregiving to manifest as relief for vulnerable groups.

\footnotetext{
${ }^{2}$ See Merriam-Webster Dictionary: https://www.merriam-webster.com/dictionary/humanity. Accessed 4 July 2020.
} 


\section{“Fragility and Vulnerability"-Who is Really Vulnerable to COVID-19?}

If this question needed just a simple answer, we could simply say "everybody", since all peoples from all walks of life are wanting as they confront the COVID-19 pandemic. The pandemic has undoubtedly demonstrated its might as a potential health threat from which no human being can convincingly claim exclusion. That is, every human being faces the danger of being harmed by COVID-19 anywhere and at any time. This makes it not an oversight to assume a priori that the pandemic has basically placed all human beings at the same potential level of health vulnerability. Though there is a great heterogeneity in the definition of vulnerability (de Groot et al. 2019), health vulnerability is generally understood to mean being exposed to potential health harm (ten Have 2016).

But considering the multidimensional implications of the concept of vulnerability, the question on vulnerability in relation to COVID-19 may be more complicated than imagined. To begin with, vulnerability is one of the most talked-about and most confusing concepts, especially in the health domain where there is the risk of closely aligning it with the specific principles of autonomy and choice vis-à-vis any potential research exploitation/harm (Kwek 2017). Besides, additional complications come from the confusion between the ontological universalization of vulnerability as an integral characteristic of a human being (Ries and Thomson 2019) and the intuitive "labelling" of some groups of people as being vulnerable (Kwek 2017). In the midst of these philosophical rigmaroles, vulnerability remains a reality of human existence, though its gravity is often conditioned in some situations by the socio-economic capability and affordability (Richard et al. 2016).

As new infections and health disasters appear across the world - as the case of COVID-19 - the concept of vulnerability is widening, thus enlarging the scope of ethical debates within healthcare systems. That is, the question of who is really vulnerable is being enlarged by the question on what exactly constitutes/determines vulnerability. For example, with COVID-19, myriad implications provoke vulnerability: biologically, +70 elders are more vulnerable; socio-culturally, black people and other minority ethnic groups like the Asiatic and the indigenous people are more vulnerable; socio-economically, people with limited income/revenue are more vulnerable; socio-politically, slum/shantytown dwellers are more vulnerable, as well as people in communitarian cultural setups. When we are fighting to prevent such a wide range of potential harm from being actualized, vulnerability is given a conditional (if ...) connotation because the harm is still in potency and will only actualize if not prevented.

This "multidimensionality" is complicating healthcare strategies to tackle vulnerability and avert its ramifications because, in this category of health vulnerability, as Henk ten Have (2016) highlighted, it is mainly the possible harm resulting from the unsuitable preventive mechanism that is at issue and not only its positive outcome. As such, the idea of vulnerability is evoked when "a person is capable of being hurt but the damage has not yet occurred, [and] this will probably happen unless appropriate measures will be taken. This conditionality generates a responsibility to take care and preventive action, if at least harm could be prevented" (ten Have 2016, 22). From this "attributive stage" of general vulnerability to health harm, we need to proceed to the "judgemental stage" by evaluating the susceptibility and/or the adaptability of individuals or groups of individuals or communities to that 
potential harm so as to determine the degree of "risk" associated to the potential harm predicted.

This level of evaluating health vulnerability opens us to health inequalities associated to various determinants - social, economic, political, cultural and environmentalthat surround our daily lives (Marmot 2020). If we put these determinants together, we get a categorization of vulnerability into physical, emotional and cognitive, and they all influence the actions, emotions, thoughts and convictions of victims, and these are all facets of healthcare challenges (Boldt 2019). These categories of vulnerability have different degrees of susceptibility and adaptability vis-à-vis COVID-19 (Xafis 2020), thereby manifesting different risks levels and necessitating some adjustments in the preventive mechanism.

It means that, while it is true, from a general view of vulnerability, that every human being is potentially vulnerable in the context of COVID-19, biological, social, economic, gender, racial and political disparities have placed some people, as a matter of fact, at higher risk. For example, emerging evidence indicates that the groups of people mentioned above are precisely at increased risk of being vulnerable to COVID-19 pandemic as they are, in fact, disproportionately affected by this disease. According to Vicki Xafis $(2020,1)$, "those individuals and groups routinely disadvantaged by the social injustice created by the misdistribution of power, money, and resources" are disproportionately impacted by this pandemic. In addition, within various disadvantaged groups and many other people, the combination of the fear of the illness caused by the pandemic and of the difficulties of following the restrictive public health measures heightens the state of the COVID-19 vulnerability (Gopichandran et al. 2020). Worst of all, as a new pandemic, its micro-biological/biomedical details are still limited.

If we could just transfer knowledge from facts about other communicable diseases that already existed (Stevenson and Riley 2004; Kakkilaya 2015), we could take as the starting point that some of the individuals with systems that resist the clinical manifestation of the potential harm perpetrated by the virus, and/or those with higher adaptability and lower susceptibility, may develop a certain degree of immunity against COVID-19 infection and reinfection. But given that no much micro-biological/biomedical information about COVID-19 is yet clear, no well-defined preventive measure is guaranteed, and vulnerable victims abound amidst the search for the accurate solution. This is necessitating a quest for a socially sanctioned non-medical preventive mechanism that can simultaneously prevent the intra-community propagation of the COVID-19 virus and the further disadvantaging of the vulnerable. We believe that if any inter-person preventive measure is to be a success, it should possess a good dosage of "care and love" for one another, putting together vulnerable citizens with asymptomatic carriers.

\section{"Vulnerable Citizens" with "Asymptomatic Carriers"—Care Ethics in the Time of COVID-19}

We have come to realize that there are diverse forms of vulnerability in the domain of healthcare (Delgado Rodriguez 2017) and diverse measures to protect the vulnerable or prevent vulnerability (Macklin 2003) and that in front of new health crisis, we need 
ethical judgement to determine those who are more at risk (ten Have 2016). Though a lot of biomedical facts are yet clear about COVID-19, the pandemic has unveiled a certain degree of the "overlapping layers" of vulnerability - both medical and social vulnerabilities. While medical vulnerability is conceived as "a feature of specific groups that triggers protection and interventions to reduce vulnerability" (Boldt 2019, 2 ), social vulnerability is "a dynamic state that reflects converging effects of a set of interacting and amplifying personal and environmental factors, which together increase an individual's susceptibility to ill health and which hampers the recovery process to normal health once ill health has occurred" (de Groot et al. 2019, 12).

In relation to the latter, it has come to light that socio-cultural stigmatization, socioeconomic disequilibrium, socio-political disadvantages and ill-weakened subsistence, putting together aging, immensely contribute to the stressful experience of the COVID19 pandemic (Yancy 2020; Nott 2020). This situation demands strategic dynamics in various preventive mechanisms since, unlike in the former where vulnerability is sought in order to protect it from exploitation (Macklin 2003; Boldt 2019), we are facing the latter where vulnerability, surrounded by various socio-ethical conditions, instead needs to be prevented from manifesting. For this reason, it has to be given a conditional evaluation: if a particular preventive measure is taken, and in a particular way, vulnerability will manifest among some particular groups; if this particular measure is not taken, vulnerability will manifest among some other groups.

All these conditionalities are determined from the degree of susceptibility and adaptability evaluated with the spirit of care ethics that tickles the moral duty of everyone to be responsible for everyone. This is because the vulnerabilities related to the COVID-19 pandemic are not just about the risks perpetrated by the disease. They also emanate from lockdowns that are not possible for many vulnerable groups, as well as from the recovery policies to take communities out of lockdown as they disproportionately affect vulnerable groups (Nott 2020). The proper management of this dynamism is still hampered by the insufficient knowledge of the micro-biological characteristics of the COVID-19 virus.

However, for reasons yet to be fully established, medical personnel are coming to the conclusion that there is a group of infected persons who are or have become asymptomatic. Dr. Anthony Fauci-director of the National Institute of Allergy and Infectious Disease, USA - affirmed that between 25 and $50 \%$ of the people infected with the COVID-19 disease are asymptomatic (Augusta Health 2020). Reading from the rate of the intra-community transmission of the COVID-19 virus around the world, health experts are further coming to the assertion that some or all of the asymptomatic people may be carriers and/or infectious (Augusta Health 2020). This group is of particular interest and concern for this article because the presence of the infectious asymptomatic individuals in our societies can complicate the coexistence of medical vulnerability and social vulnerability within our daily interrelationships. Medical vulnerability (ill health) is often exacerbated by social vulnerability due to lack of income support and access to healthcare, and it risks developing into a vicious cycle (Rogers 2014).

The main worry of this article is to determine how to avert the eventuality of this stage of the COVID-19 pandemic, should "the vulnerable" closely cohabit with "the asymptomatic carriers" who can unknowingly be disguised propagators of the virus. Should this be true, then asymptomatic carriers of the COVID-19 virus can be 
potentially "dangerous" for the whole society, especially as its propagation has proven to be human-to-human. Since this knowledge is yet to be clearer, it implies that with the day-to-day experience of COVID-19, the global society is exposed, among many, to three degrees of risk:

- The risk of ignorantly living with infectious asymptomatic individuals who unknowingly incubate and propagate the virus

- The risk of aggravating the health situation of the susceptible individuals - like the +70 elders and ill-weakened-by unknowingly exposing them to closer contact with the infectious asymptomatic carriers

- The risk of amplifying "the vulnerable susceptibility level" of the people with weaker socio-economic capabilities by worsening their health situation amidst lack of good health and healthcare adequacy.

Though antiviral immunity can be enforced with induced antigens to prevent transmission and reinfection (Klimpel 1996), only clinical evaluation can validate if it works with COVID-19. While still in doubts, it might be a precautionary necessity that in battling with COVID-19 as an infectious pandemic, except otherwise established, we should proceed a priori on the bases that almost all those who are not clinically infected are potential asymptomatic carriers who might be naturally disguised infection agents. In other words, without any stigmatization, we need to intuitively consider anybodyourselves inclusive - as a potential virus carrier capable of infecting others, since we might unknowingly be infectious asymptomatic carriers. Given that vulnerability, as already hinted above, is primordially associated with possible harm (ten Have 2016), the socio-economic, socio-cultural, socio-political and socio-psychological "shadow pandemics" of COVID-19 - both on the infected and the affected - might be devastating in case this foresight is overlooked.

Let us imagine a scenario where infectious asymptomatic people happen to be working in long-term care homes, or sharing a communitarian family life with elderly people, or living in poorer overcrowded households/shantytowns or frequenting overcrowded public areas and transportation systems. The risks involved in any of these possibilities would have necessitated massive/frequent immunity/asymptomatic screening, but individual and societal social and economic vulnerabilities cannot permit this. However, the World Health Organization (WHO) came up with a catalogue of preventive measures: "Wash your hands frequently; Maintain social distancing; If you have fever, cough and difficulty breathing, seek medical care early; Stay informed and follow advice given by your healthcare provider" (Xafis 2020, 2).

As effective as these measures are, they are potential sources of vulnerability. Some socio-cultural, socio-economic and socio-biological/physical conditions do not warrant these measures: social distancing is impossible in high population density setups; and many "poorer" people lack access to adequate healthcare. This means that, whether asymptomatic or not, many individuals and/or groups simply cannot protect themselves and others or from others, and they will find themselves in "shadow pandemics" (unnecessary fallouts), given that human health, as already highlighted above, does not only refer to physical illness. This is where applied ethics, in its capacity as socioethical mode, motif and motive (Motilal 2010), transfers the insight from care ethics into the domain of bioethics as applied care ethics (Sander-Staudt 2020). That is, since 
there is significant social stigmatization associated with COVID-19 (Gopichandran et al. 2020), applied care ethics assures that any particular preventive measure implemented, the way it is implemented, and the extent of its effects do not provoke any form of vulnerability to COVID-19 or to its shadow pandemics. We can better consider antiCOVID-19 policies from care ethics perspective.

From care ethics perspective as applied care ethics to prevent the rampant interperson/intra-community propagation of COVID-19, public health authorities have commonly been emphasizing, inter alia, social/physical distancing and/or the wearing of face/mouth masks. Despite the socio-epistemological cacophony provoked by the concept of "social distancing", public health experts use it with simple understanding as a preventive measure against a communicable pandemic like COVID-19. In that light, "social distancing", as well as "physical distancing", is simply understood as "a term applied to certain actions that are taken by Public Health officials to stop or slow down the spread of a highly contagious disease" (Public Health Department 2020, 1). Notwithstanding this clarification, the concept of "distancing"- be it social or physical - can still be misinterpreted as "escaping from the other" instead of simply understanding it with the spirit of care as a preventive caution to "distance ourselves".

This socio-epistemological controversy on the concept of social distancing can be another potential source of stigmatization or xenophobia. Coupling it with the sociological grounding of the concept of "social distancing" which principally links it to "the behavioral complex of activities and their derivatives" (Hall 1968, 83), we are prompted not to take this controversy for granted. According to the Centers for Disease Control, USA, "approximately 50\% of preventable deaths are related to behavioral functions" (Scrimshaw et al. 2001, 9). This is why it is clearer, in the words of Philip Ethington that "the concept of social distance needs to be refashioned [...], but precisely how it must be refashioned is a question that I think we are only beginning to see" (Ethington 1997, 2).

To be clearer, proficient vulnerability analysis indicates that a policy of social distancing is neither possible nor effective for and among many groups. This article advocates that we should address this potential lapse with the effective use of the spirit of care ethics. In this light, the insight from care ethics lures us into asking what alternative policy might complement social distancing and help to reduce risks. The mandatory wearing of face masks is viewed as one candidate of such policies. We do, of course, recognize that vulnerable groups might still face some challenges like the non-access to masks and/or the educational deficits about their effective use. But these challenges are comparatively easier and cheaper to be handled, and it is worth doing so, given the vitality/necessity. "Face/mouth masks" wearing is the mechanism in which conscience plays a great role to complement "I" and "Others"- egoism and altruismin preventing the propagation of COVID-19.

Therefore, to manifest care and responsibility in the time of this crisis, "we should all act as if we could be carriers of the virus" (Augusta Health 2020). From the trust and confidence that we have in our health systems (Gopichandran et al. 2020), we believe with health experts that the COVID-19 virus is predominantly propagated through oral and/or nasal "moist droplets". It thus goes without saying that "mask" wearing is a more plausible intra-community COVID-19 preventive measure upheld by the common interdependence strength of care/love. This strength, according to Peterson and Seligman $(2004,17)$, “contributes to various fulfilments that constitute, the good life 
for oneself and for others". In this light, care ethics surfaces as the antithesis of individualistic ethics and nourishes the togetherness capable of resisting disintegration, even in times of hardship, as posits communitarianism (Metz 2013).

The choice of obligating the wearing of face/mouth masks in public places to prevent the propagation of COVID-19 shows how humans, as social beings, rely on each other for survival, especially during crisis of this magnitude. Above all,

[t]his pandemic has exposed how reliant we all are on each other, how the health of the disadvantaged impacts on the advantaged, how events in one country impact on lives in others, how economies are impacted by the health of the people whose labour they rely on and on the health of those excluded from the labour market, and how we can only fight some battles standing united. (Xafis 2020, 3)

Besides the fact that mask wearing is more practicable, especially in social setups where physical distancing might be difficult, it is also a preventive measure in which individuals foremostly prevent the "self" from contaminating the "others". Also, it carries no risk of stigmatization if everybody in the society is obliged to wear. But it can be a call for stigmatization if it is allowed on choice (facultative) because the people who will decide to be wearing it may be stigmatized as the infected. If every society proficiently enforces this measure as serious as necessary, each citizen will play part in preventing the contamination of others. With this dynamism, everyone must have been the "carer" of everyone, as holds the philosophical theory of communitarianism (Metz 2013). At the end, everyone will protect the other (duty to care) and will in turn be protected by others (right to care) against COVID-19, and the whole society will be protected to a greater extent, for no one might be saved until everyone is saved.

\section{Conclusion-Discussion}

Though the infection vulnerability of COVID-19 is seemingly universal, there is a certain degree of inequalities in its resulting risks, thereby necessitating equity in healthcare (anti-COVID-19) intervention. By equity in healthcare, we mean a strategy in which the trajectory or the intensity of healthcare intervention is determined by "need" or susceptibility (Richard et al. 2016). In the case of COVID-19, the higher the susceptibility ratio — + 70 elders, ill-weakened and street dwellers - the higher the need for a rapid and intensive intervention strategy. But COVID-19's vulnerability has multi-dimensionally escalated beyond physical to include social, cultural, economic, political ... With the spirit of care ethics, we credit the obligatory mask wearing as a more "care-oriented" method that simultaneously enforces (1) the prevention of the "inter-human" propagation of COVID-19; (2) the "inter-person" responsibility of social care within our societies and (3) the reduction of the related stigmatization crisis.

We acknowledge the ethical implications of "obligating/mandating" a measure on the citizenry as it tempers with the exercise of personal liberty. But we also acknowledge that when health crisis like the COVID-19 pandemic strikes, health authorities take the responsibility of evoking some swift and liberty limiting yet proportionate, population-based healthcare measures/strategies to mitigate its propagation (Upshur 2003; Evans 2020). Mouth/face mask wearing is one of such anti-COVID-19 measures 
judged necessary enough to be enforced, especially at this stage when lockdown relaxations are rendering human interaction more complicated and intensifying vulnerability. Judging from the socio-ethical perspective, this measure satisfies necessary/ fundamental ethical requirements: first, it satisfies "the harm principle" in that it is undoubtful that this measure will reduce the potential measurable harm to others should the disease or its exposure go unchecked; and, second, it satisfies "the least-restrictivemeans principle" (Upshur 2003, 393) in that it is a gradual add-up measure to complement the relaxation of strict confinement and distancing measures and to ease social circulation and interaction.

Moreover, this measure can minimize possible impressions of estrangement that could develop in people with some social handicaps like the visually impaired, together with citizens in some socio-cultural setups where distancing measures are difficult. With this scenario at hand, the search for the way forward necessitates a wider dimension of ideas: first, because the solution pathfinding mechanism is still at the stage of "preventive research" (Scrimshaw et al. 2001, 6); second, because the responsibility of such a global health encounter is necessarily interdisciplinary (Dine 2020); and third, because applied global bioethics needs to exhibit intercultural, interdisciplinary and international content and context (Chukwuneke et al. 2014; Benatar and Singer 2000). If this dexterity endowed in mask wearing is overlooked, the consequences of the pandemic may be exacerbated by "shadow pandemics"-both on the infected and the affected - caused by various behavioural derivatives like stress, stigmatization, abandonment, loneliness and rejection, just to name but these few.

Acknowledgements The author is very grateful for the insightful review process.

\section{Compliance with Ethical Standards}

Conflict of interest The author declares that he has no conflict of interest.

\section{References}

Augusta Health. 2020. COVID-19 Asymptomatic Carriers and Antibody Tests. Augusta Health, 23 April 2020. https:/www.augustahealth.com/health-focused/covid-19-asymptomatic-carriers-and-antibody-tests. Accessed 4 August 2020.

Benatar, Solomon, and Peter Singer. 2000. A new look at international research ethics. BMJ 321: 824-826. https://doi.org/10.1136/bmj.321.7264.824.

Boldt, Joachim. 2019. The concept of vulnerability in medical ethics and philosophy. Philosophy, Ethics, and Humanities in Medicine 14: 6. https://doi.org/10.1186/s13010-019-0075-6.

Chukwuneke, Felix N., O. Umeora, J. Maduabuchi, and N. Egbunike. 2014. Global bioethics and culture in a pluralistic world: how does culture influence bioethics in Africa? Annals of Medical and Health Sciences Research 4 (5): 672-675. https://doi.org/10.4103/2141-9248.141495.

de Groot, Nynke, Gouke J. Bonsel, Erwin Birnie, and Nicole B. Valentine. 2019. Towards a universal concept of vulnerability: broadening the evidence from the elderly to perinatal health using a Delphi approach. Plos One 14 (2): 1932-6203. https://doi.org/10.1371/journal.pone.0212633.

De Panfilis, Ludovica, Silvia Di Leo, Carlo Peruselli, Luca Ghirotto and Silvia Tanzi. 2019. "I go into crisis when ...": ethics of care and moral dilemmas in palliative care. BMC Palliative Care 18: 70. https://doi. org/10.1186/s12904-019-1453-2.

Delgado Rodriguez, Janet. 2017. The relevance of the ethics of vulnerability in bioethics. Les ateliers de l'éthique / The Ethics Forum 12 (2-3): 154-179. https://doi.org/10.7202/1051280ar. 
Dine, Charles Biradzem. 2016. Bioethics: glory to science, dream to Africans (Bioethics \& Cultural Diversity). MA Thesis, Université du Québec à Rimouski. http://semaphore.uqar.ca/id/eprint/1169. Accessed 4 August 2020.

Dine, Charles Biradzem. 2020. 'Responsible' in Global Health management: ingenious interdisciplinarity as relief to the epistemological complexity of transdisciplinarity. Les chantiers de l'intervention en sciences humaines 5: 62-72. http://labrri.net/parution-du-nouveau-volume-de-la-revue-chantiers-de-linterventionen-sciences-humaines-interdisciplinarite-pratique-et-action-professionnelle/. Accessed 4 August 2020.

Ethington, Philip J. 1997. The intellectual construction of "social distance": toward a recovery of Georg Simmel's social geometry. Cybergeo, document 30. https://doi.org/10.4000/cybergeo.227.

Evans, Nicholas. 2020. Covid-19: the ethics of clinical research in quarantine. BMJ 369: m2060. https://doi. org/10.1136/bmj.m2060.

Gopichandran, Vijayaprasad, Sudharshini Subramaniam, and Maria Jusler Kalsingh. 2020. COVID-19 pandemic: a litmus test of trust in the health system. Asian Bioethics Review 12 (2): 213-221. https://doi.org/10.1007/s41649-020-00122-6.

Hall, Edward. 1968. Proximics. Current Anthropology 9 (2/3): 83-108. https://doi.org/10.1086/200975.

Held, Virginia. 2007. The ethics of care. In The Oxford Handbook of Ethical Theory, ed. David Copp. New York, NY: Oxford University Press. https://doi.org/10.1093/oxfordhb/9780195325911.003.0020.

Kakkilaya, B. Srinivas. 2015. Immunity. Malaria Site, 9 March 2015. https://www.malariasite. com/immunity/. Accessed 20 November 2019.

Klimpel, Gary R. 1996. Immune defenses. In Medical microbiology, $4^{\text {th }}$ edition, ed. S. Baron. Galveston, TX: University of Texas Medical Branch at Galveston. https://www.ncbi.nlm.nih.gov/books/NBK8423/. Accessed 2 May 2020.

Kroeger-Mappes, Joy. 1994. The ethic of care vis-à-vis the ethic of rights: a problem for contemporary moral theory. HYPATIA 9 (3): 108-131. https://doi.org/10.1111/j.1527-2001.1994.tb00452.x.

Kwek, Adrian. 2017. The indispensability of labelled groups to vulnerability in bioethics. Bioethics 31: 674 682. https://doi.org/10.1111/bioe.12379.

Macklin, Ruth. 2003. Bioethics, vulnerability, and protection. Bioethics 17 (5-6): 472-486. https://doi. org/10.1111/1467-8519.00362.

Marmot, Michael. 2020. Health equity in England: the Marmot review 10 years on. BMJ 368: m693. https://oi.org/10.1136/bmj.m693.

Metz, Thaddeus. 2013. The western ethic of care or an afro-communitarian ethic? Specifying the right relational morality. Journal of Global Ethics 9 (1): 77-92. https://doi.org/10.1080/17449626.2012.756421.

Motilal, Shashi. 2010. Applied ethics and human rights: Conceptual analysis and contextual applications. New Delhi: Anthem Press.

Nair, Indira. 2005. Ethics of care. In Encyclopedia of Science, Technology, and Ethics, ed. Carl Mitcham. Detroit, MI: Macmillan Reference USA.

Nott, David. 2020. The COVID-19 response for vulnerable people in places affected by conflict and humanitarian crises. Lancet 395 (10236): 1532-1533. https://doi.org/10.1016/s0140-6736(20)31036-9.

Okano, Yayo. 2016. Why has the ethics of care become an issue of global concern? International Journal of Japanese Sociology 25 (1): 85-99. https://doi.org/10.1111/ijjs.12048.

Peterson, Christopher, and Martin E. P. Seligman. 2004. Character Strengths and Virtues: A Handbook and Classification. New York, NY: Oxford University Press / Washington, DC: American Psychological Association.

Public Health Department. 2020. Information about Social Distancing, Fact sheet. Santa Clara Valley Health \& Hospital System, County of Santa Clara. https://www.cidrap.umn.edu/sites/default/files/public/php/185 /185_factsheet_social_distancing.pdf. Accessed 4 July 2020.

Richard, Lauralie, John Furler, Konstancja Densley, Jeannie Haggerty, Grant Russell, Jean-Frederic Levesque, and Jane Gunn. 2016. Equity of access to primary healthcare for vulnerable populations: the IMPACT international online survey of innovations. International Journal for Equity in Health 15: 64. https://doi. org/10.1186/s12939-016-0351-7.

Ries, Nola M., and Michael Thomson. 2019. Bioethics and universal vulnerability: exploring the ethics and practices of research participation. Medical Law Review 28 (2): 293-316. https://doi.org/10.1093 /medlaw/fwz026.

Sander-Staudt, Maureen. 2020. Care Ethics. In Internet Encyclopedia of Philosophy, ed. James Fieser, and Bradley Dowden. https://www.iep.utm.edu/care-eth/\#H10. Accessed 18 May 2020.

Scrimshaw, Susan C., LuAnn White, and Jeffrey P. Koplan. 2001. The meaning and value of prevention research. Public Health Reports 116 (suppl 1): 4-9. https://doi.org/10.1093/phr/116.s1.4.

Stevenson, Mary, and Eleanor Riley. 2004. Innate immunity to malaria. Nature Review Immunology 4: 169180. https://doi.org/10.1038/nri1311. 
ten Have, Henk. 2016. Vulnerability: challenging Bioethics. New Yok, NY: Routledge.

Upshur, Ross. 2003. The ethics of quarantine. AMA Journal of Ethics 5 (11): 393-395. https://doi.org/10.1001 /virtualmentor.2003.5.11.msoc1-0311.

van Dijke, Jolanda, Inge van Nistelrooij, Pien Bos, and Joachim Duyndam. 2019. Care ethics: an ethics of empathy? Nursing Ethics 26 (5): 1282-1291. https://doi.org/10.1177/0969733018761172.

Rogers, Wendy. 2014. Vulnerability and Bioethics. In Vulnerability: New Essays in Ethics and Feminist Philosophy, ed. Catriona Mackenzie, Wendy Rogers, and Susan Dodds. Oxford University Press. https://doi.org/10.1093/acprof:oso/9780199316649.003.0003.

OHCHR, and WHO. 2008. The Right to Health, Fact Sheet No. 31. Geneva: Office of the High Commissioner for Human Rights, and World Health Organization. https://www.who.int/gender-equityrights/knowledge/right-to-health-factsheet/en/. Accessed 2 Sept 2017.

Xafis, Vicki. 2020. 'What is inconvenient for you is life-saving for me': how health inequities are playing out during the COVID-19 pandemic. Asian Bioethics Review 12 (2): 223-234. https://doi.org/10.1007 /s41649-020-00119-1.

Yancy, Clyde. 2020. COVID-19 and African Americans. JAMA 353 (19): 1891-1892. https://doi.org/10.1001 jjama.2020.6548.

Publisher's Note Springer Nature remains neutral with regard to jurisdictional claims in published maps and institutional affiliations. 